\title{
BRAF Rearrangement Analysis
}

National Cancer Institute

\section{Source}

National Cancer Institute. BRAF Rearrangement Analysis. NCI Thesaurus. Code C157188.

A procedure used to detect and identify rearrang ements involving the BRAF gene. 